\title{
On-line Bayesian Cramer-Rao Bounds for OFDM Slowly Varying Rayleigh Multi-path Channel Estimation
}

\author{
Hussein Hijazi and Laurent Ros \\ GIPSA-lab, Image and Signal Department \\ BP 46 - 38402 Saint Martin d'Hères - FRANCE \\ E-mail: hussein.hijazi@gipsa-lab.inpg.fr, laurent.ros@gipsa-lab.inpg.fr \\ Tel: +33 (0)4 76827178 and Fax: +33 (0)4 76826384
}

\begin{abstract}
The on-line Bayesian Cramer-Rao (BCRB) lower bound for the dynamic estimation of a time-varying multi-path Rayleigh channel in 4-QAM OFDM system is considered. In case of negligible channel variation within one symbol and delay related information, true BCRB for data-aided (DA) context, and two closed-form expressions for non-data aided (NDA) context are derived.
\end{abstract}

Index Terms-Bayesian Cramer-Rao Bound, OFDM, Rayleigh complex gains.

\section{INTRODUCTION}

In case of Orthogonal Frequency Division Multiplexing (OFDM) mobile communication systems, dynamic estimation of frequency selective and time-varying channel is a fundamental function [1]. Channel estimation can be summarized to estimate of certain physical propagation parameters, such as multi-path delays and multi-path complex gains [2] [3] [5]. In Radio-Frequency transmission with slow variations, the number of paths and time delays can be easily obtained [2] [3], since the delays are quasi-invariant over a large number of symbols. Assuming the availability of delay related information, the question that arises is of the ultimate accuracy that can be achieved in channel estimation operations. Tools to approach this problem are available from the parameters estimation theory [11] in form of the Cramer-Rao Bounds (CRBs), which give fundamental lower limits of the Mean Square Error (MSE) achievable by any unbiased estimator. A Modified CRB (MCRB), easier to evaluate than the Standard CRB (SCRB), has been introduced in [6] [7]. The MCRB proves useful when, in addition to the parameter to be estimated, the observed data also depend on other unwanted parameters. More recently, the problem of deriving CRBs, suited to time-varying parameters, has been addressed throughout the Bayesian context. In [9], the authors propose a general framework for deriving analytical expression of on-line CRBs. In [10], the authors introduce a new asymptotic bound, namely the Asymptotic Bayesian CRB (ABCRB), for the mono-carrier phase estimation problem and Non-Data-Aided (NDA) scenario. This bound is closer to the classical BCRB than the Modified BCRB (MBCRB) and is easier to evaluate than BCRB. In this contribution we investigate the $\mathrm{BCRB}$ related to the estimation of the complex gains of a rayleigh channel, assuming negligible time variation within one OFDM symbol (usual context for OFDM modulation). Explicit expressions of the BCRB and its variants, MBCRB and $\mathrm{ABCRB}$, are provided for NDA and DA 4-QAM on-line scenarios.

Notations : $[\mathbf{x}]_{k}$ denotes the $k$ th entry of the vector $\mathbf{x}$, and $[\mathbf{X}]_{k, m}$ the $[k, m]$ th entry of the matrix $\mathbf{X}$. As in matlab $\mathbb{B}$, $\mathbf{X}\left[k_{1}: k_{2}, m_{1}: m_{2}\right]$ is a submatrix extracted from rows $k_{1}$ to $k_{2}$ and from columns $m_{1}$ to $m_{2}$ of $\mathbf{X}$. $\operatorname{diag}\{\mathbf{x}\}$ is a diagonal matrix with $\mathbf{x}$ on its diagonal, $\operatorname{diag}\{\mathbf{X}\}$ is a vector whose elements are the elements of the diagonal of $\mathbf{X}$ and blkdiag $\{\mathbf{X}, \mathbf{Y}\}$ is a block diagonal matrix with the matrices $\mathbf{X}$ and $\mathbf{Y}$ on its diagonal. $\mathrm{E}_{x, y}[\cdot]$ denotes the expectation over $x$ and $y . J_{0}(\cdot)$ is the zeroth-order Bessel function of the first kind. $\nabla_{\mathbf{x}}$ and $\Delta_{\mathbf{y}}^{\mathbf{x}}$ represent the first and the second-order partial derivatives operator i.e., $\nabla_{\mathbf{x}}=\left[\frac{\partial}{\partial x_{1}}, \ldots, \frac{\partial}{\partial x_{N}}\right]^{T}$ and $\Delta_{\mathbf{y}}^{\mathbf{x}}=\nabla_{\mathbf{y}}^{*} \nabla_{\mathbf{x}}^{T}$.

\section{SyStem MOdel}

Consider an OFDM system with $\mathrm{N}$ sub-carriers, and a cyclic prefix length $N_{g}$. The duration of an OFDM symbol is $T=v T_{s}$, where $T_{s}$ is the sampling time and $v=N+N_{g}$. Let $\mathbf{x}_{(n)}=\left[x_{(n)}\left[-\frac{N}{2}\right], x_{(n)}\left[-\frac{N}{2}+1\right], \ldots, x_{(n)}\left[\frac{N}{2}-1\right]\right]^{T}$ be the $n$th transmitted OFDM symbol, where $\left\{x_{(n)}[b]\right\}$ are normalized 4-QAM symbols. After transmission over a multipath Rayleigh channel, the $n$th received OFDM symbol $\mathbf{y}_{(n)}=$ $\left[y_{(n)}\left[-\frac{N}{2}\right], y_{(n)}\left[-\frac{N}{2}+1\right], \ldots, y_{(n)}\left[\frac{N}{2}-1\right]\right]^{T}$ is given by [2] [3]:

$$
\mathbf{y}_{(n)}=\mathbf{H}_{(n)} \mathbf{x}_{(n)}+\mathbf{w}_{(n)}
$$

where $\mathbf{w}_{(n)}$ is a $N \times 1$ zero-mean complex Gaussian noise vector with covariance matrix $\sigma^{2} \mathbf{I}_{N}$, and $\mathbf{H}_{(n)}$ is a $N \times N$ diagonal matrix with diagonal elements given by:

$$
\left[\mathbf{H}_{(n)}\right]_{k, k}=\frac{1}{N} \sum_{l=1}^{L}\left[\alpha_{l}^{(n)} \times e^{-j 2 \pi\left(\frac{k-1}{N}-\frac{1}{2}\right) \tau_{l}}\right]
$$

$L$ is the total number of propagation paths, $\alpha_{l}$ is the $l$ th complex gain of variance $\sigma_{\alpha_{l}}^{2}$ (with $\sum_{l=1}^{L} \sigma_{\alpha_{l}}^{2}=1$ ), and $\tau_{l} \times T_{s}$ is the $l$ th delay ( $\tau_{l}$ is not necessarily an integer, but $\tau_{L}<N_{g}$ ). The $L$ individual elements of $\left\{\alpha_{l}^{(n)}\right\}$ are uncorrellated with respect to each other. They are wide-sense stationary narrowband complex Gaussian processes, with the so-called Jakes' power spectrum [12] with Doppler frequency $f_{d}$. It means 
that $\alpha_{l}^{(n)}$ are correlated complex gaussian variables with zeromeans and correlation coefficients given by:

$$
\mathrm{R}_{\alpha_{l}}^{(p)}=\mathrm{E}\left[\alpha_{l}^{(n)} \alpha_{l}^{(n-p)^{H}}\right]=\sigma_{\alpha_{l}}^{2} J_{0}\left(2 \pi f_{d} T p\right)
$$

Using (2), the observation model in (1) for the $n$th OFDM symbol can be re-written as:

$$
\mathbf{y}_{(n)}=\operatorname{diag}\left\{\mathbf{x}_{(n)}\right\} \mathbf{F} \boldsymbol{\alpha}_{(n)}+\mathbf{w}_{(n)}
$$

where $\boldsymbol{\alpha}_{(n)}=\left[\alpha_{1}^{(n)}, \ldots, \alpha_{L}^{(n)}\right]^{T}$ is a $L \times 1$ vector and $\mathbf{F}$ is the $N \times L$ Fourier matrix defined by:

$$
[\mathbf{F}]_{k, l}=e^{-j 2 \pi\left(\frac{k-1}{N}-\frac{1}{2}\right) \tau_{l}}
$$

\section{BAYESIAN CRAMER-RAO BOUNDS (BCRBs)}

In this section, we present a general formulation of the BCRB related to the estimation of the multi-path complex gains. In NDA context, we derive a closed-form expression of a BCRB, i.e., the Asymptotic BCRB or the Modified BCRB. In DA context, we deduce the computation of the true BCRB from the computation of the MBCRB in NDA. $\hat{\boldsymbol{\alpha}}(\mathbf{y})$ denote an unbiased estimator of $\boldsymbol{\alpha}=\left[\boldsymbol{\alpha}_{(1)}{ }^{T}, \ldots, \boldsymbol{\alpha}_{(K)}\right]^{T}$ using the set of measurements $\mathbf{y}=\left[\mathbf{y}_{(1)}{ }^{T}, \ldots, \mathbf{y}_{(K)}{ }^{T}\right]^{T}$. In the on-line scenario, the receiver estimates $\boldsymbol{\alpha}_{(n)}$ based on the current and previous observations only, i.e., $\mathbf{y}=\left[\mathbf{y}_{(1)}{ }^{T}, \ldots, \mathbf{y}_{(n)}{ }^{T}\right]^{T}$.

\section{A. Bayesian Cramer-Rao bound}

The BCRB is particularly suited when a priori information is available. The BCRB has been proposed in [11] such that:

$$
\mathrm{E}_{\mathbf{y}, \boldsymbol{\alpha}}\left[(\hat{\boldsymbol{\alpha}}(\mathbf{y})-\boldsymbol{\alpha})(\hat{\boldsymbol{\alpha}}(\mathbf{y})-\boldsymbol{\alpha})^{H}\right] \geq \operatorname{BCRB}(\boldsymbol{\alpha})
$$

The $\mathrm{BCRB}^{1}$ is the inverse of the Bayesian Information Matrix (BIM) B, which can be written as:

$$
\mathbf{B}=\mathrm{E}_{\boldsymbol{\alpha}}[\mathbf{F}(\boldsymbol{\alpha})]+\mathrm{E}_{\boldsymbol{\alpha}}\left[-\Delta_{\boldsymbol{\alpha}}^{\boldsymbol{\alpha}} \ln (p(\boldsymbol{\alpha}))\right]
$$

where $p(\boldsymbol{\alpha})$ is the prior probability density function (pdf) and $\mathbf{F}(\boldsymbol{\alpha})$ is the Fisher Information Matrix (FIM) defined as:

$$
\mathbf{F}(\boldsymbol{\alpha})=\mathrm{E}_{\mathbf{y} \mid \boldsymbol{\alpha}}\left[-\Delta_{\boldsymbol{\alpha}}^{\boldsymbol{\alpha}} \ln (p(\mathbf{y} \mid \boldsymbol{\alpha}))\right]
$$

where $p(\mathbf{y} \mid \boldsymbol{\alpha})$ is the conditional pdf of $\mathbf{y}$ given $\boldsymbol{\alpha}$. The on-line BCRB associated to observation vector $\mathbf{y}=$ $\left[\mathbf{y}_{(1)}{ }^{T}, \ldots, \mathbf{y}_{(K)}^{T}\right]^{T}$ will be obtained [10] by:

$$
\operatorname{BCRB}\left(\boldsymbol{\alpha}_{(K)}\right)_{\text {online }}=\operatorname{Tr}\left(\mathbf{B C R B}(\boldsymbol{\alpha})_{[i(K), i(K)]}\right)
$$

where $i(n)$ is a sequence of indices defined by $i(n)=1+(n-1) L: n L$ with $n \in[1, K]$. This definition (9) will stand for the closed form BCRBs.

1) Computation of $\mathbf{E}_{\boldsymbol{\alpha}}\left[-\Delta_{\boldsymbol{\alpha}}^{\boldsymbol{\alpha}} \ln (p(\boldsymbol{\alpha}))\right]: \boldsymbol{\alpha}$ is a complex Gaussian vector with zero mean and covariance matrix $\mathbf{R}_{\boldsymbol{\alpha}}=$ $E\left\{\boldsymbol{\alpha} \boldsymbol{\alpha}^{H}\right\}$ of size $K L \times K L$ defined as:

$$
\left[\mathbf{R}_{\boldsymbol{\alpha}}\right]_{i(l, p), i\left(l^{\prime}, p^{\prime}\right)}=\left\{\begin{array}{clc}
\mathbf{R}_{\alpha_{l}}^{\left(p-p^{\prime}\right)} & \text { for } l^{\prime}=l \in[1, L] & p, p^{\prime} \in[0, K-1] \\
0 & \text { for } l^{\prime} \neq l
\end{array}\right.
$$

\footnotetext{
${ }^{1}$ We recall that the Standard Cramer-Rao Bound (SCRB) is the inverse of the Fisher Information Matrix $\mathbf{F}(\boldsymbol{\alpha})$ (a priori information is not used).
}

where $i(l, p)=1+(l-1)+p L$ and $\mathbf{R}_{\alpha_{l}}^{(p)}$ is defined in (3). For example, if $K=L=2$ then, $\mathbf{R}_{\boldsymbol{\alpha}}$ is given by:

$$
\mathbf{R}_{\alpha}=\left[\begin{array}{cccc}
\mathbf{R}_{\alpha_{1}}^{(0)} & 0 & \mathbf{R}_{\alpha_{1}}^{(-1)} & 0 \\
0 & \mathbf{R}_{\alpha_{2}}^{(0)} & 0 & \mathbf{R}_{\alpha_{2}}^{(-1)} \\
\mathbf{R}_{\alpha_{1}}^{(1)} & 0 & \mathbf{R}_{\alpha_{1}}^{(0)} & 0 \\
0 & \mathbf{R}_{\alpha_{2}}^{(1)} & 0 & \mathbf{R}_{\alpha_{2}}^{(0)}
\end{array}\right]
$$

Thus, the pdf $p(\boldsymbol{\alpha})$ is defined as:

$$
p(\boldsymbol{\alpha})=\frac{1}{\left|\pi \mathbf{R}_{\boldsymbol{\alpha}}\right|} e^{-\boldsymbol{\alpha}^{H} \mathbf{R}_{\alpha}^{-1} \boldsymbol{\alpha}}
$$

Taking the second derivative of the natural logarithm of (12) with respect to $\boldsymbol{\alpha}$ and making the expectation over $\boldsymbol{\alpha}$, hence:

$$
\mathrm{E}_{\boldsymbol{\alpha}}\left[-\Delta_{\boldsymbol{\alpha}}^{\boldsymbol{\alpha}} \ln (p(\boldsymbol{\alpha}))\right]=\mathbf{R}_{\boldsymbol{\alpha}}^{-1}
$$

2) Computation of $\mathbf{E}_{\boldsymbol{\alpha}}[\mathbf{F}(\boldsymbol{\alpha})]$ : Using the whiteness of the noise and the independence of the transmitted OFDM symbols, one obtains from the observation model in (4) that:

$$
\Delta_{\boldsymbol{\alpha}}^{\boldsymbol{\alpha}} \ln (p(\mathbf{y} \mid \boldsymbol{\alpha}))=\sum_{n=1}^{K} \Delta_{\boldsymbol{\alpha}}^{\boldsymbol{\alpha}} \ln \left(p\left(\mathbf{y}_{(n)} \mid \boldsymbol{\alpha}_{(n)}\right)\right)
$$

Each term of the sum (14) is a $K L \times K L$ block diagonal matrix with only one nonzero $L \times L$ block matrix, namely:

$$
\Delta_{\boldsymbol{\alpha}}^{\boldsymbol{\alpha}} \ln \left(p\left(\mathbf{y}_{(n)} \mid \boldsymbol{\alpha}_{(n)}\right)\right)_{[i(n), i(n)]}=\Delta_{\boldsymbol{\alpha}_{(n)}}^{\boldsymbol{\alpha}_{(n)}} \ln \left(p\left(\mathbf{y}_{(n)} \mid \boldsymbol{\alpha}_{(n)}\right)\right)
$$

As a direct consequence, $\Delta_{\boldsymbol{\alpha}}^{\boldsymbol{\alpha}} \ln (p(\mathbf{y} \mid \boldsymbol{\alpha}))$ is a block diagonal matrix with the $n$th diagonal block given by (15). Moreover, because of the circularity of the observation noise, the expectation of (15) with respect to $\mathbf{y}_{(n)}$ and $\boldsymbol{\alpha}_{(n)}$ does not depend on $\boldsymbol{\alpha}_{(n)}$. One then obtains:

$$
\mathrm{E}_{\boldsymbol{\alpha}}[\mathbf{F}(\boldsymbol{\alpha})]=\operatorname{blkdiag}\{\mathbf{J}, \mathbf{J}, \ldots, \mathbf{J}\}
$$

where $\mathbf{J}$ is a $L \times L$ matrix defined as:

$$
\mathbf{J}=\mathrm{E}_{\mathbf{y}, \boldsymbol{\alpha}}\left[-\Delta_{\boldsymbol{\alpha}_{(n)}}^{\boldsymbol{\alpha}_{(n)}} \ln \left(p\left(\mathbf{y}_{(n)} \mid \boldsymbol{\alpha}_{(n)}\right)\right)\right]
$$

The log-likelihood function in (17) can be expanded as:

$\ln \left(p\left(\mathbf{y}_{(n)} \mid \boldsymbol{\alpha}_{(n)}\right)\right)=\ln \left(\sum_{\mathbf{x}_{(n)}} p\left(\mathbf{y}_{(n)} \mid \mathbf{x}_{(n)}, \boldsymbol{\alpha}_{(n)}\right) p\left(\mathbf{x}_{(n)}\right)\right)$

The vector $\mathbf{y}_{(n)}$ for given $\mathbf{x}_{(n)}$ and $\boldsymbol{\alpha}_{(n)}$ is complex Gaussian with mean vector $\mathbf{m}_{(n)}=\operatorname{diag}\left\{\mathbf{x}_{(n)}\right\} \mathbf{F} \boldsymbol{\alpha}_{(n)}$ and covariance matrix $\sigma^{2} \mathbf{I}_{N}$. Thus, the conditional pdf is :

$p\left(\mathbf{y}_{(n)} \mid \mathbf{x}_{(n)}, \boldsymbol{\alpha}_{(n)}\right)=\frac{1}{\left|\pi \sigma^{2} \mathbf{I}_{N}\right|} e^{-\frac{1}{\sigma^{2}}\left(\mathbf{y}_{(n)}-\mathbf{m}_{(n)}\right)^{H}\left(\mathbf{y}_{(n)}-\mathbf{m}_{(n)}\right)}$

Since each element of the vector $\mathbf{m}_{(n)}$ depends on only one element of $\mathbf{x}_{(n)}$ then, using the Gaussian nature of the noise and the equiprobability of the normalized QAM symbols, one finds (see Appendix A) that:

$$
\begin{gathered}
\ln \left(p\left(\mathbf{y}_{(n)} \mid \boldsymbol{\alpha}_{(n)}\right)\right)=\ln \left[\frac{1}{\left|\pi \sigma^{2} \mathbf{I}_{N}\right|} e^{-\frac{1}{\sigma^{2}}\left(\mathbf{y}_{(n)}^{H} \mathbf{y}_{(n)}+\boldsymbol{\alpha}_{(n)}^{H} \mathbf{F}^{H} \mathbf{F} \boldsymbol{\alpha}_{(n)}\right)}\right. \\
\left.\prod_{k=1}^{N} \cosh \left(\frac{\sqrt{2}}{\sigma^{2}} \operatorname{Re}\left(a_{n}(k)\right)\right) \cosh \left(\frac{\sqrt{2}}{\sigma^{2}} \operatorname{Im}\left(a_{n}(k)\right)\right)\right]
\end{gathered}
$$


where $a_{n}(k)=\left[\mathbf{y}_{(n)}\right]_{k}^{*} \mathbf{g}_{k}^{T} \boldsymbol{\alpha}_{(n)}$ and $\mathbf{g}_{k}^{T}$ is the $k$ th row of the matrix F. The result of the second derivative of (20) with respect to $\boldsymbol{\alpha}_{(n)}$ is given by:

$\Delta_{\boldsymbol{\alpha}_{(n)}}^{\boldsymbol{\alpha}_{(n)}} \ln \left(p\left(\mathbf{y}_{(n)} \mid \boldsymbol{\alpha}_{(n)}\right)\right)=-\frac{1}{\sigma^{2}} \mathbf{F}^{H} \mathbf{F}+\sum_{k=1}^{N}\left[\frac{1}{2 \sigma^{4}}\left[\mathbf{y}_{(n)}\right]_{k}\left[\mathbf{y}_{(n)}\right]_{k}^{*}\right.$ $\left.\mathbf{g}_{k}^{*} \mathbf{g}_{k}^{T}\left(2-\tanh ^{2}\left(\frac{\sqrt{2}}{\sigma^{2}} \operatorname{Re}\left(a_{n}(k)\right)\right)-\tanh ^{2}\left(\frac{\sqrt{2}}{\sigma^{2}} \operatorname{Im}\left(a_{n}(k)\right)\right)\right)\right]$

The expectation of (21) with respect to $\mathbf{y}_{(n)} \mid \boldsymbol{\alpha}_{(n)}$ does not have any simple analytical solution. Hence, we have to resort to either numerical integration methods or some approximations.

In conlusion, the BRCB of the Rayleigh multi-path channel gains estimation problem, for an OFDM observation model, takes the following form :

$$
\mathbf{B C R B}(\boldsymbol{\alpha})=\left(\operatorname{blkdiag}\{\mathbf{J}, \mathbf{J}, \ldots, \mathbf{J}\}+\mathbf{R}_{\boldsymbol{\alpha}}^{-1}\right)^{-1}
$$

where the matrix $\mathbf{J}$ obtained from (17) and (21) is difficult to compute analytically. In the following, we present both the high-SNR and the low-SNR approximations of the BCRB, in order to compute the Asymptotic BCRB as defined in [10].

\section{B. Asymptotic BCRB}

1) High-SNR BCRB Asymptote: From the definition of BIM (7), only the first term (i.e., $\left.\mathrm{E}_{\boldsymbol{\alpha}}[\mathbf{F}(\boldsymbol{\alpha})]\right)$ depends on the $\mathrm{SNR}$, which is fully characterized by $\mathbf{J}$. Hence, we focus on the behavior of $\mathbf{J}$. At high SNR (i.e., $\sigma^{2} \rightarrow 0$ ), the tanh-function in (21) can be approximated as: $\tanh \left(\frac{\sqrt{2}}{\sigma^{2}} x\right) \approx \operatorname{sgn}(x)$. Hence, we obtain the high-SNR asymptote of $\mathbf{J}$, which is:

$$
\mathbf{J}_{h}=\frac{1}{\sigma^{2}} \mathbf{F}^{H} \mathbf{F}
$$

2) Low-SNR BCRB Asymptote: Following the same reasoning as before, at low SNR (i.e., $\sigma^{2} \rightarrow+\infty$ ), we have $\tanh (x) \approx x$ arround $x=0$. Hence, we obtain:

$$
\begin{gathered}
\Delta_{\boldsymbol{\alpha}_{(n)}}^{\boldsymbol{\alpha}_{(n)}} \ln \left(p\left(\mathbf{y}_{(n)} \mid \boldsymbol{\alpha}_{(n)}\right)\right) \approx-\frac{1}{\sigma^{2}} \mathbf{F}^{H} \mathbf{F}+ \\
\sum_{k=1}^{N}\left[\frac{1}{\sigma^{8}}\left[\mathbf{y}_{(n)}\right]_{k}\left[\mathbf{y}_{(n)}\right]_{k}^{*} \mathbf{g}_{k}^{*} \mathbf{g}_{k}^{T}\left(\sigma^{4}-a_{n}(k) a_{n}^{*}(k)\right)\right]
\end{gathered}
$$

Plugging (24) into (17), we obtain the low-SNR asymptote of $\mathbf{J}$, which is (see Appendix B):

$$
\mathbf{J}_{l}=\left(\frac{\beta}{\sigma^{4}}+\frac{8 \beta^{2}}{\sigma^{6}}+\frac{6 \beta^{3}}{\sigma^{8}}\right) \mathbf{F}^{H} \mathbf{F}
$$

where $\beta=\sum_{l=1}^{L} \sigma_{\alpha_{l}}^{2}$ is the total channel energy.

We can then combine the low- and high- SNR BRCB asymptotes from (23), (25) and (22) to define the Asymptotic $\mathrm{BCRB}(\mathrm{ABCRB})$ as :

$$
\operatorname{ABCRB}(\boldsymbol{\alpha})=\left(\operatorname{blkdiag}\left\{\mathbf{J}_{m i n}, \ldots, \mathbf{J}_{m i n}\right\}+\mathbf{R}_{\boldsymbol{\alpha}}^{-1}\right)^{-1}
$$

where

$$
\begin{gathered}
\mathbf{J}_{\min }=\min \left(v_{l}, v_{h}\right) \cdot \mathbf{F}^{H} \mathbf{F} \\
v_{l}=\frac{\beta}{\sigma^{4}}+\frac{8 \beta^{2}}{\sigma^{6}}+\frac{6 \beta^{3}}{\sigma^{8}} \quad \text { and } \quad v_{h}=\frac{1}{\sigma^{2}}
\end{gathered}
$$

This ABRCB is well a lower bound of the MSE (see appendix $\mathrm{C}$ ).

\section{Modified BCRB}

The analytical computation of $\mathbf{F}(\boldsymbol{\alpha})$ is quite tedious in case of NDA context because of the OFDM symbols $\mathbf{x}=$ $\left[\mathbf{x}_{(1)}^{T}, \ldots, \mathbf{x}_{(K)}{ }^{T}\right]^{T}$, which are "nuisance parameters". In order to circumvent this kind of problem, a Modified BCRB (MBCRB) has been proposed in [6]. This MBCRB is the inverse of the following information matrix:

$$
\mathbf{C}=\mathrm{E}_{\boldsymbol{\alpha}}[\mathbf{G}(\boldsymbol{\alpha})]+\mathrm{E}_{\boldsymbol{\alpha}}\left[-\Delta_{\boldsymbol{\alpha}}^{\boldsymbol{\alpha}} \ln (p(\boldsymbol{\alpha}))\right]
$$

where $\mathbf{G}(\boldsymbol{\alpha})$ is the modified FIM defined as:

$$
\mathbf{G}(\boldsymbol{\alpha})=\mathrm{E}_{\mathbf{x}} \mathrm{E}_{\mathbf{y} \mid \mathbf{x}, \boldsymbol{\alpha}}\left[-\Delta_{\boldsymbol{\alpha}}^{\boldsymbol{\alpha}} \ln (p(\mathbf{y} \mid \mathbf{x}, \boldsymbol{\alpha}))\right]
$$

Hence, following the same reasoning as before, we have:

$$
\mathrm{E}_{\boldsymbol{\alpha}}[\mathbf{G}(\boldsymbol{\alpha})]=\operatorname{blkdiag}\left\{\mathbf{J}_{m}, \mathbf{J}_{m}, \ldots, \mathbf{J}_{m}\right\}
$$

where $\mathbf{J}_{m}$ is a $L \times L$ matrix defined as:

$$
\mathbf{J}_{m}=\mathrm{E}_{\mathbf{y}, \mathbf{x}, \boldsymbol{\alpha}}\left[-\Delta_{\boldsymbol{\alpha}_{(n)}}^{\boldsymbol{\alpha}_{(n)}} \ln \left(p\left(\mathbf{y}_{(n)} \mid \mathbf{x}_{(n)}, \boldsymbol{\alpha}_{(n)}\right)\right)\right]
$$

By taking the second derivative of the natural logarithm $(\ln )$ of (19) with respect to $\boldsymbol{\alpha}_{(n)}$, one easily obtains that:

$$
\mathbf{J}_{m}=\mathrm{E}_{\mathbf{x}}\left[\frac{1}{\sigma^{2}} \mathbf{F}^{H} \operatorname{diag}\left\{\mathbf{x}_{(n)}^{H}\right\} \operatorname{diag}\left\{\mathbf{x}_{(n)}\right\} \mathbf{F}\right]
$$

which leads to:

$$
\mathbf{J}_{m}=\frac{1}{\sigma^{2}} \mathbf{F}^{H} \mathbf{F}
$$

since the QAM-symbols are normalized. The MBCRB for the estimation of $\boldsymbol{\alpha}$ is given by:

$$
\operatorname{MBCRB}(\boldsymbol{\alpha})=\left(\operatorname{blkdiag}\left\{\mathbf{J}_{m}, \mathbf{J}_{m}, \ldots, \mathbf{J}_{m}\right\}+\mathbf{R}_{\boldsymbol{\alpha}}^{-1}\right)^{-1}
$$

We see that $\mathbf{J}_{h}=\mathbf{J}_{m}$ hence, the high-SNR asymptote of the $\mathrm{BCRB}$ is equal to the MBCRB. This corroborates the result of [8] for a scalar parameter in non-Bayesian case. Moreover, In Appendix C, we show that:

$$
\operatorname{MBCRB}(\alpha) \leq \operatorname{ABCRB}(\alpha) \leq \operatorname{BCRB}(\alpha)
$$

This corroborates the result derived in [10] for a mono-carrier phase estimation problem.

Notice that the term $\Delta_{\boldsymbol{\alpha}_{(n)}}^{\boldsymbol{\alpha}_{(n)}} \ln \left(p\left(\mathbf{y}_{(n)} \mid \mathbf{x}_{(n)}, \boldsymbol{\alpha}_{(n)}\right)\right)=$ $-\frac{1}{\sigma^{2}} \mathbf{F}^{H} \mathbf{F}$ does not depend on the transmitted data sequence $\mathbf{x}_{(n)}$. Hence, the true BCRB in data-aided (DA) context is equal to the MBCRB in non-data-aided (NDA) context.

\section{DISCUSSION}

In this section, we illustrate the behavior of the previous bounds for the complex gains estimation. A 4QAM-OFDM system with normalized symbols, $N=128$ subcarriers and $N_{g}=\frac{N}{8}$ is used. The normalized Rayleigh channel contains $L=6$ paths and others parameters given in [3] [4].

Fig. 1 presents the on-line BCRB (evaluated by MonteCarlo trials), $\mathrm{ABCRB}$ and $\mathrm{MBCRB}$ versus $\operatorname{SNR}\left(=\frac{1}{\sigma^{2}}\right)$, for a block-observation length $K=20$ and a normalized Doppler frequency $f_{d} T=10^{-3}$. We plot also as reference the Standard 


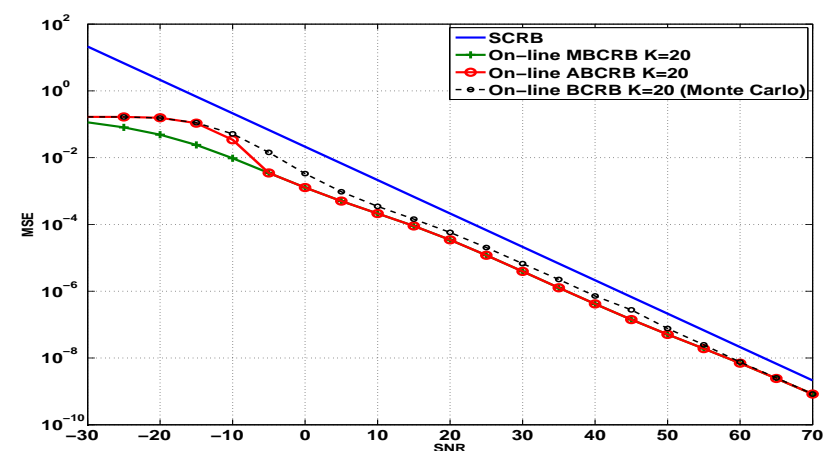

Fig. 1. SCRB and BCRBs vs SNR for $f_{d} T=10^{-3}$ and $\mathrm{K}=20$

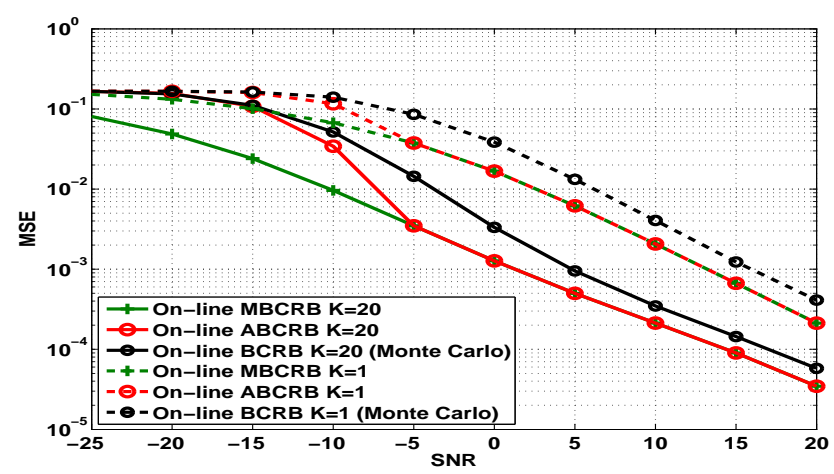

Fig. 2. BCRBs vs SNR for $f_{d} T=10^{-3}$ and two different observation lengths $K=1$ and $K=20$

$\mathrm{CRB}\left(\mathrm{SCRB}{ }^{2}\right.$ ) corresponding to the case where the a priori information is not used. We observe that the ABCRB and the MBCRB are less than SCRB since the prior information of the complex gains is taken into account. We also verify that $M B C R B \leq A B C R B \leq B C R B$, as proved in Appendix C. At high SNR, the MBCRB and the ABCRB are very close, as predicted by our theoretical analysis.

Fig. 2 is a zoom of Fig. 1 for the BRCBs with $K=20$. It also shows the BCRBs for a block-observation length $K=1$, i.e., when the estimator uses only the current symbol instead of the current and past symbols. First, we can measure the potential performance improvement for Data Aided (MBCRB) versus Non Data Aided (BRCB) scenarios. Secondly, we can measure the great potential gain obtained in taking into account the past information $(K=20$ versus $K=1)$.

Fig. 3 emphasizes this last point in presenting the on-line ABRCB (versus SNR) for $f_{d} T=10^{-4}$ and various observation lengths $K=1,5,20,60$. We notice that the estimation can be greatly improved when the number of observations $K$ increases, since the estimator takes into account more past informations. It is then interesting to have an idea of the observation length requisite to acquire major part of the gain, which is the aim of Fig. 4.

Fig. 4 presents the on-line ABCRB versus time index $K$ for different normalized Doppler frequencies $f_{d} T\left(10^{-5}\right.$ to $5.10^{-3}$ ) and $S N R=10 \mathrm{~dB}$. When the number of observations increases, the bound decreases and converges to an asymptote. The estimation gain using past symbols is naturally

\footnotetext{
${ }^{2} \mathrm{SCRB}$ is computed as the inverse of $J_{m}$ in equation (34). It is the true SCRB in DA context or the Modified SCRB in NDA context.
}

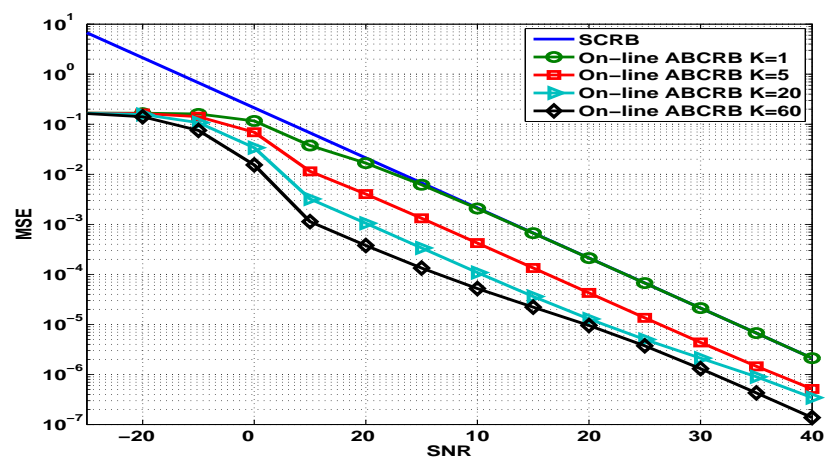

Fig. 3. ABCRB vs SNR for $f_{d} T=10^{-4}$ and various $K$

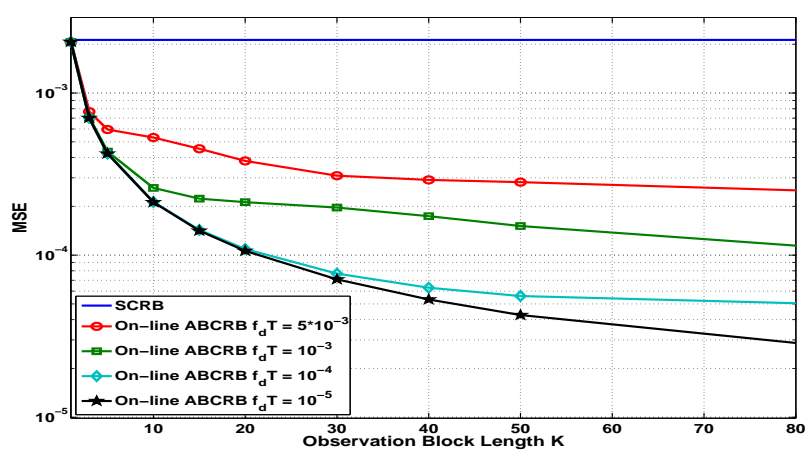

Fig. 4. BCRB vs observation length, for $S N R=10 \mathrm{~dB}$

more significant for slow channel variations (low $f_{d} T$ ).

\section{CONCLUSion}

In this contibution, we have derived analytical expressions of BCRB for the estimation of Rayleigh multi-path channel complex gains with slow time variations. These bounds are useful when analyzing the performance of complex gains estimators in DA and NDA contexts and in on-line scenarios. Moreover, they permit to measure the benefit of using past OFDM symbols for channel estimation process, whereas most methods use only the current symbol [1].

\section{APPENDIX A}

DERIVATION OF EXPRESSION (20) AND (21)

Plugging (19) into (18), we obtain:

$$
\begin{gathered}
\ln \left(p\left(\mathbf{y}_{(n)} \mid \boldsymbol{\alpha}_{(n)}\right)\right)=-\frac{1}{\sigma^{2}}\left(\mathbf{y}_{(n)}^{H} \mathbf{y}_{(n)}+\mathbf{m}_{(n)}^{H} \mathbf{m}_{(n)}\right)+ \\
\ln \left(\frac{p\left(\mathbf{x}_{(n)}\right)}{\left|\pi \sigma^{2} \mathbf{I}_{N}\right|} \sum_{\mathbf{x}_{(n)}} e^{\left.\frac{2}{\sigma^{2}} \operatorname{Re}\left(\mathbf{y}_{(n)}^{H} \mathbf{m}_{(n)}\right)\right)}\right.
\end{gathered}
$$

since the 4QAM-symbols are equiprobable (i.e., $p\left(\mathbf{x}_{(n)}\right)=$ $\left.\frac{1}{4^{N}}\right)$. However, $\mathbf{m}_{(n)}=\operatorname{diag}\left\{\mathbf{x}_{(n)}\right\} \mathbf{F} \boldsymbol{\alpha}_{(n)}$ then, $\mathbf{y}_{(n)}^{H} \mathbf{m}_{(n)}=$ $\sum_{k=1}^{N} a_{n}(k)\left[\mathbf{x}_{(n)}\right]_{k}$ where $a_{n}(k)$ is defined in section III part A. Hence, one obtains:

$$
\sum_{\mathbf{x}_{(n)}} e^{\frac{2}{\sigma^{2}} \operatorname{Re}\left(\mathbf{y}_{(n)}^{H} \mathbf{m}_{(n)}\right)}=\prod_{k=1}^{N}\left(\sum_{\left[\mathbf{x}_{(n)}\right]_{k}} e^{\frac{2}{\sigma^{2}} \operatorname{Re}\left(a_{n}(k)\left[\mathbf{x}_{(n)}\right]_{k}\right)}\right)
$$


Since $\left[\mathbf{x}_{(n)}\right]_{k}=\frac{1}{\sqrt{2}}( \pm 1 \pm j)$ (4QAM-symbol), we obtain:

$$
\begin{aligned}
& \sum_{\left[\mathbf{x}_{(n)}\right]_{k}} e^{\frac{2}{\sigma^{2}} \operatorname{Re}\left(a_{n}(k)\left[\mathbf{x}_{(n)}\right]_{k}\right)}= \\
& 4 \cosh \left(\frac{\sqrt{2}}{\sigma^{2}} \operatorname{Re}\left(a_{n}(k)\right)\right) \cosh \left(\frac{\sqrt{2}}{\sigma^{2}} \operatorname{Im}\left(a_{n}(k)\right)\right)
\end{aligned}
$$

Inserting this result into (37), we obtain the expression in (20). Taking the second derivative of (20) with respect to $\boldsymbol{\alpha}_{(n)}$ and using $\nabla_{\boldsymbol{\alpha}_{(n)}} \operatorname{Re}\left(a_{n}(k)\right)=\frac{1}{2}\left[\mathbf{y}_{(n)}\right]_{k}^{*} \mathbf{g}_{k}$ and $\nabla_{\boldsymbol{\alpha}_{(n)}} \operatorname{Im}\left(a_{n}(k)\right)=$ $\frac{1}{2 j}\left[\mathbf{y}_{(n)}\right]_{k}^{*} \mathbf{g}_{k}$, we obtain finally the expression in (21).

\section{APPENDIX B}

EVALUATION OF $\mathbf{J}_{l}$ IN (25)

Inserting the definition of $a_{n}(k)$ into (24) and plugging the result into (17), one obtains:

$$
\begin{gathered}
\mathbf{J}_{l}=\frac{1}{\sigma^{2}} \mathbf{F}^{H} \mathbf{F}-\frac{1}{\sigma^{4}} \sum_{k=1}^{N} \mathbf{g}_{k}^{*} \mathrm{E}_{\boldsymbol{\alpha}} \mathrm{E}_{\mathbf{y} \mid \boldsymbol{\alpha}}\left[\left[\mathbf{y}_{(n)}\right]_{k}\left[\mathbf{y}_{(n)}\right]_{k}^{*}\right] \mathbf{g}_{k}^{T} \\
+\frac{1}{\sigma^{8}} \sum_{k=1}^{N} \mathbf{g}_{k}^{*} \mathbf{g}_{k}^{T} \mathrm{E}_{\boldsymbol{\alpha}}\left[\boldsymbol{\alpha}_{(n)} \boldsymbol{\alpha}_{(n)}{ }^{H} \mathbf{g}_{k}^{*} \mathrm{E}_{\mathbf{y} \mid \boldsymbol{\alpha}}\left[\left(\left[\mathbf{y}_{(n)}\right]_{k}\left[\mathbf{y}_{(n)}\right]_{k}^{*}\right)^{2}\right]\right] \mathbf{g}_{k}^{T}
\end{gathered}
$$

Using that $\left[\mathbf{y}_{(n)}\right]_{k}=\left[\mathbf{x}_{(n)}\right]_{k} \mathbf{g}_{k}^{T} \boldsymbol{\alpha}_{(n)}+\left[\mathbf{w}_{(n)}\right]_{k}$, the independance between the QAM-symbols and the noise, and these results below:

$$
\begin{aligned}
& \mathrm{E}_{\left[\mathbf{x}_{(n)}\right]_{k}}\left[\left[\mathbf{x}_{(n)}\right]_{k}^{2}\right]=\mathrm{E}_{\left[\mathbf{w}_{(n)}\right]_{k}}\left[\left[\mathbf{w}_{(n)}\right]_{k}^{2}\right]=0 \\
& \mathrm{E}_{\left[\mathbf{w}_{(n)}\right]_{k}}\left[\left[\mathbf{w}_{(n)}\right]_{k}^{2}\left[\mathbf{w}_{(n)}\right]_{k}^{* 2}\right]=2 \sigma^{4}
\end{aligned}
$$

we obtain:

$$
\begin{gathered}
\mathrm{E}_{\mathbf{y} \mid \boldsymbol{\alpha}}\left[\left[\mathbf{y}_{(n)}\right]_{k}\left[\mathbf{y}_{(n)}\right]_{k}^{*}\right]=\mathbf{g}_{k}^{T} \boldsymbol{\alpha}_{(n)} \boldsymbol{\alpha}_{(n)}^{H} \mathbf{g}_{k}^{*}+\sigma^{2} \\
\mathrm{E}_{\mathbf{y} \mid \boldsymbol{\alpha}\left[\left(\left[\mathbf{y}_{(n)}\right]_{k}\left[\mathbf{y}_{(n)}\right]_{k}^{*}\right)^{2}\right]}=2 \sigma^{4}+4 \sigma^{2} \mathbf{g}_{k}^{T} \boldsymbol{\alpha}_{(n)} \boldsymbol{\alpha}_{(n)}^{H} \mathbf{g}_{k}^{*} \\
+\mathbf{g}_{k}^{T} \boldsymbol{\alpha}_{(n)} \boldsymbol{\alpha}_{(n)}^{H} \mathbf{g}_{k}^{*} \mathbf{g}_{k}^{T} \boldsymbol{\alpha}_{(n)} \boldsymbol{\alpha}_{(n)}^{H} \mathbf{g}_{k}^{*}
\end{gathered}
$$

Hence, $\mathbf{J}_{l}$ becomes:

$\mathbf{J}_{l}=\frac{1}{\sigma^{4}} \sum_{k=1}^{N} \mathbf{V}_{k} \mathbf{D} \mathbf{V}_{k}+\frac{4}{\sigma^{6}} \sum_{k=1}^{N} \mathbf{V}_{k} \mathrm{E}_{\boldsymbol{\alpha}}\left[\mathbf{T}_{1}\right] \mathbf{V}_{k}+\frac{1}{\sigma^{8}} \sum_{k=1}^{N} \mathbf{V}_{k} \mathrm{E}_{\boldsymbol{\alpha}}\left[\mathbf{T}_{2}\right] \mathbf{V}_{k}$

where $\mathbf{V}_{k}=\mathbf{g}_{k}^{*} \mathbf{g}_{k}^{T}, \mathbf{T}_{1}=\boldsymbol{\alpha}_{(n)} \boldsymbol{\alpha}_{(n)}^{H} \mathbf{V}_{k} \boldsymbol{\alpha}_{(n)} \boldsymbol{\alpha}_{(n)}^{H}, \mathbf{T}_{2}=$ $\boldsymbol{\alpha}_{(n)} \boldsymbol{\alpha}_{(n)}^{H} \mathbf{V}_{k} \boldsymbol{\alpha}_{(n)} \boldsymbol{\alpha}_{(n)}^{H} \mathbf{V}_{k} \boldsymbol{\alpha}_{(n)} \boldsymbol{\alpha}_{(n)}^{H}$ and $\mathbf{D}=\mathrm{E}_{\boldsymbol{\alpha}}\left[\boldsymbol{\alpha}_{(n)} \boldsymbol{\alpha}_{(n)}^{H}\right]=$ $\operatorname{diag}\left\{\sigma_{\alpha_{1}}^{2}, \ldots, \sigma_{\alpha_{L}}^{2}\right\}$. The elements of $\mathbf{T}_{1}$ and $\mathbf{T}_{2}$ are given by:

$$
\begin{aligned}
{\left[\mathbf{T}_{1}\right]_{l, l^{\prime}}=} & \sum_{l_{1}=1}^{L} \sum_{l_{2}=1}^{L}\left[\mathbf{V}_{k}\right]_{l 1, l 2}\left[\boldsymbol{\alpha}_{(n)}\right]_{l}\left[\boldsymbol{\alpha}_{(n)}\right]_{l_{2}}\left[\boldsymbol{\alpha}_{(n)}\right]_{l^{\prime}}^{*}\left[\boldsymbol{\alpha}_{(n)}\right]_{l_{1}}^{*} \\
{\left[\mathbf{T}_{2}\right]_{l, l^{\prime}}=} & \sum_{l_{1}=1}^{L} \sum_{l_{2}=1}^{L} \sum_{l_{3}=1}^{L} \sum_{l_{4}=1}^{L}\left[\mathbf{V}_{k}\right]_{l 1, l 2}\left[\mathbf{V}_{k}\right]_{l 3, l 4} \\
& {\left[\boldsymbol{\alpha}_{(n)}\right]_{l}\left[\boldsymbol{\alpha}_{(n)}\right]_{l_{2}}\left[\boldsymbol{\alpha}_{(n)}\right]_{l_{4}}\left[\boldsymbol{\alpha}_{(n)}\right]_{l^{\prime}}^{*}\left[\boldsymbol{\alpha}_{(n)}\right]_{l_{1}}^{*}\left[\boldsymbol{\alpha}_{(n)}\right]_{l_{3}}^{*} }
\end{aligned}
$$

Using that $\mathrm{E}_{\left[\mathbf{c}_{(n)}\right]_{l}}\left[\left[\mathbf{c}_{(n)}\right]_{l}^{2}\right]=0$ and the definitions of 4 th and 6th order moments for complex gaussian variables, we obtain:

$$
\begin{aligned}
\mathrm{E}_{\boldsymbol{\alpha}}\left[\mathbf{T}_{1}\right]= & \mathbf{D V} \mathbf{V}_{k} \mathbf{D}+\operatorname{Tr}\left(\mathbf{V}_{k} \mathbf{D}\right) \mathbf{D} \\
\mathrm{E}_{\boldsymbol{\alpha}}\left[\mathbf{T}_{2}\right]= & 2 \mathbf{D V}_{k} \mathbf{D} \mathbf{V}_{k} \mathbf{D}+2 \operatorname{Tr}\left(\mathbf{V}_{k} \mathbf{D}\right) \mathbf{D} \mathbf{V}_{k} \mathbf{D} \\
& +\operatorname{Tr}\left(\mathbf{V}_{k} \mathbf{D} \mathbf{V}_{k} \mathbf{D}\right) \mathbf{D}+\left(\operatorname{Tr}\left(\mathbf{V}_{k} \mathbf{D}\right)\right)^{2} \mathbf{D}
\end{aligned}
$$

Using that $\mathbf{g}_{k}^{T} \mathbf{D g}_{k}^{*}=\operatorname{Tr}\left(\mathbf{V}_{k} \mathbf{D}\right)=\sum_{l=1}^{L} \sigma_{\alpha_{l}}^{2}=\beta$, $\operatorname{Tr}\left(\mathbf{V}_{k} \mathbf{D} \mathbf{V}_{k} \mathbf{D}\right)=\beta^{2}, \mathbf{D V}_{k} \mathbf{D} \mathbf{V}_{k} \mathbf{D}=\beta \mathbf{D V} \mathbf{V}_{k} \mathbf{D}$, and inserting these results into (43), we obtain the expression of $\mathbf{J}_{l}$ in (25).

\section{APPENDIX C \\ PROOF OF THE INEQUALITY (36)}

¿From the definition of $\mathbf{J}_{\text {min }}$, we have $\mathbf{J}_{\text {min }} \leq \mathbf{J}_{m}=\mathbf{J}_{h}$ and then we have the first inequality in (36), i.e., $\operatorname{MBCRB}(\boldsymbol{\alpha}) \leq$ $\operatorname{ABCRB}(\boldsymbol{\alpha})$. To prove the second inequality in (36), we have to show that $\mathbf{J} \leq \mathbf{J}_{\min }$. The $\tanh ^{2}(x)$ function is tangent to the curve $y=x^{2}$ at $x=0$ and has $y=1$ as horizontal asymptote. Hence, we can write, for every $x \geq 0$, these two properties below:

$$
\tanh ^{2}(x) \leq 1 \text { and } \tanh ^{2}(x) \leq x^{2}
$$

Using these two properties, we obtain from (21) that:

$$
\begin{aligned}
\Delta_{\boldsymbol{\alpha}_{(n)}}^{\boldsymbol{\alpha}_{(n)}} \ln \left(p\left(\mathbf{y}_{(n)} \mid \boldsymbol{\alpha}_{(n)}\right)\right) & \geq-\frac{1}{\sigma^{2}} \mathbf{F}^{H} \mathbf{F} \\
\Delta_{\boldsymbol{\alpha}_{(n)}}^{\boldsymbol{\alpha}_{(n)}} & \ln \left(p\left(\mathbf{y}_{(n)} \mid \boldsymbol{\alpha}_{(n)}\right)\right) \geq-\frac{1}{\sigma^{2}} \mathbf{F}^{H} \mathbf{F}+ \\
& \sum_{k=1}^{N}\left[\frac{1}{\sigma^{8}}\left[\mathbf{y}_{(n)}\right]_{k}\left[\mathbf{y}_{(n)}\right]_{k}^{*} \mathbf{g}_{k}^{*} \mathbf{g}_{k}^{T}\left(\sigma^{4}-a_{n}(k) a_{n}^{*}(k)\right)\right]
\end{aligned}
$$

Substituting (47) and (48) in (17), we obtain:

$$
\mathbf{J} \leq \mathbf{J}_{h} \text { and } \mathbf{J} \leq \mathbf{J}_{l}
$$

Hence, we have $\mathbf{J} \leq \mathbf{J}_{\min }$, and consequently the second inequality in (36), i.e., $\mathbf{A B C R B}(\boldsymbol{\alpha}) \leq \mathbf{B C R B}(\boldsymbol{\alpha})$.

\section{REFERENCES}

[1] M. Hsieh and C. Wei, "Channel estimation for OFDM systems based on comb-type pilot arrangement in frequency selective fading channels" in IEEE Trans. Consumer Electron., vol.44, no. 1, Feb. 1998.

[2] B. Yang, K. B. Letaief, R. S. Cheng and Z. Cao, "Channel estimation for OFDM transmisson in mutipath fading channels based on parametric channel modeling" in IEEE Trans. Commun., vol. 49, no. 3, pp. 467-479, March 2001.

[3] H. Hijazi and L. Ros, "Polynomial estimation of time-varying multi-path gains with intercarrier interference mitigation in OFDM systems" in IEEE Trans. Vehicular Techno., to be appeared in Jan. 2009.

[4] H. Hijazi and L. Ros, "Time-varying Channel Complex Gains Estimation and ICI Suppression in OFDM Systems" in IEEE Global Communications Conf., Washington, USA, Nov. 2007.

[5] E. Simon, L. Ros and K. Raoof,"Synchronization over rapidly timevarying multipath channel for CDMA downlink RAKE receivers in TimeDivision mode", in IEEE Trans. Vehicular Tech., vol. 56. no. 4, Jul. 2007

[6] B. Z. Bobrovosky, E. Mayer-Wolf and M. Zakai, "Some classes of global Cramer-Rao bounds" in Ann. Statistics, vol. 15, pp. 1421-1438, 1987.

[7] A. D'Andrea, U. Mengali and R. Reggiannini, "The modified Cramer-Rao bound and its application to synchronization problems" in IEEE Trans. Commun., vol. 42, pp. 1391-1399, Apr. 1994.

[8] M. Moeneclaey, "On the true and the modified Cramer-Rao bounds for the estimation of a scalar parameter in the presence of nuisance parameters" in IEEE Trans. Com., vol. 46, no. 11, pp. 1536-1544, Nov. 1998.

[9] P. Tichavsky, C. H. Murachvik and A. Nehorai, "Posterior CramerRao bound for discrete-time nonlinear filtering" in IEEE Trans. Signal Processing, vol. 46, pp. 1386-1396, May. 1998.

[10] S. Bay, C. Herzet, J. M. Brossier, J. P. Barbot and B. Geller, "Analytic and asymptotic analysis of Bayesian Cramer-Rao bound for dynamical phase offset estimation" in IEEE Trans. Signal Processing, vol. 56, pp. 61-70, Jan. 2008.

[11] H. L. Van Trees, Detection, estimation, and modulation theory: Part I, Wiley, New York, 1968.

[12] W. C. Jakes, Microwave Mobile Communications. IEEE Press, 1983. 